\title{
Design and Implementation of Multi Agent Simulation Library MasCUDA for Parallel Processing with GPU
}

\author{
Akira Ohiwa \\ Graduate School of Science and Engineering, \\ Doshisha University \\ 1-3 Miyakotani, Tatara, Kyoto Japan \\ $+81-774-65-6978$
}

\author{
Hirohide Haga \\ Graduate School of Science and Engineering, \\ Doshisha University \\ 1-3 Miyakotani, Tatara, Kyoto Japan \\ +81-774-65-6978 \\ hhaga@mail.doshisha.ac.jp
}

\begin{abstract}
This paper presents the design and implementation of parallel processing support library, primary for multi-agent simulation with GPU (Graphical Processing Unit). GPU provides highly parallel processing environment. However, in order to develop software for GPU, high level skill and knowledge of GPU, parallel processing and GPU architecture are required, and these requirements sometimes disturb to use GPU for specific application development. In this article we will provide the library for GPU programming named MasCUDA. Users can develop their own application by their familiar language such as Ruby. GPU specific programming is hidden by MasCUDA and users need not to understand the detail of GPU programming. Our experimental evaluation proved that MasCUDA accelerates the execution speed more than 5,000 times faster than Ruby program and the number of source code with MasCUDA is approximately half of GPU specific language.
\end{abstract}

\section{CCS Concepts}

- Computing methodologies $\rightarrow$ Modeling and simulation $\rightarrow$ Simulation types and techniques $\rightarrow$ Massively parallel and high-performance simulations;

- Software and its engineering $\rightarrow$ Domain specific languages.

\section{Keywords}

Parallel processing; GPU; Domain specific languages; LLVM; Ruby.

\section{INTRODUCTION}

In this article, the design and implementation of multi-agent simulation (Multi-Agent Simulation) library MasCUDA for GPGPU is presented. MasCUDA is a library for developing multi agent simulation software using GPU.

In recent years, MAS [1] has been receiving much attention as a technique for developing the models of various phenomenon and simulating physical and social phenomenon. MAS system consists

Permission to make digital or hard copies of all or part of this work for personal or classroom use is granted without fee provided that copies are not made or distributed for profit or commercial advantage and that copies bear this notice and the full citation on the first page. Copyrights for components of this work owned by others than ACM must be honored. Abstracting with credit is permitted. To copy otherwise, or republish, to post on servers or to redistribute to lists, requires prior specific permission and/or a fee. Request permissions from Permissions@acm.org.

EEET 2019, September 25-27, 2019, Penang, Malaysia

(C) 2019 Association for Computing Machinery.

ACM ISBN 978-1-4503-7214-5/19/09 ..\$15.00

DOI: https://doi.org/10.1145/3362752.3362770 of multiple, sometimes more than thousands, agents that act autonomously based on their own criteria, and the space in which they exist. A key feature of MAS is the ability to observe phenomena resulting from the mutual interaction between micro phenomena that occur from mutual interaction between agents and macro phenomena over the entire space. However, the scale of the model to be simulated may make it difficult to achieve realistic processing speeds when running MAS only on a CPU. This is because the number of agents becomes larger, so sequential execution on a CPU would require a huge amount of time. One of the most promising methods of resolving this problem is the usage of parallel processing. For this reason, the highly parallelism of the Graphics Processing Unit (GPU) receives particular attention [2]. However, programming using GPU requires extensive knowledge of the architecture and programming model of GPUs, or in other words, high technical skills and knowledge of GPU architecture and its programming model is required. In addition, while NVIDIA Corporation provides CUDA [3] as a framework for GPU programming, its kernel language is CUDA-C, which is a variant of $\mathrm{C}$ programming language. As a result, programming with GPU requires many low-level descriptions for device control and other purposes, all of which disturb the usage of GPU by developers.

Therefore, we start the design and implementation of MAS library named MasCUDA. MasCUDA is a library for Ruby [4] that enables MAS to be easily implemented without being familiar with GPGPU programming, which enables developers to focus their efforts on implementing MAS algorithms. MasCUDA also provides a visualization function that plays an important role in MAS

\section{RELATED WORKS ON USING GPGPU FROM OTHER LANGUAGES}

There are several studies about the use of GPGPU from other languages with the aim of resolving the problems described above. These include PyCUDA [5], PyOpenCL [6], and JCUDA [7]. These studies aim to facilitate GPGPU programming by using the Python or Java to enable the use of CUDA from a language other than $\mathrm{C}$ or $\mathrm{C}++$. However, these systems, while making it easier to use CUDA, suffer themselves from several problems. PyCUDA, for example, has the advantage of omitting memory management, but when using CUDA with a dynamic typing language like Python, it must be kept in mind that Parallel Thread Extension (PTX), the CUDA assembly language, is a typed language. This means that Python's key feature of omitting typing cannot be used and that types must be specified. JCUDA runs on JavaVM equipped with garbage collection (GC), but since GC is separate from CUDA memory management, the problem of the need for memory management in CUDA remains. In addition, both 
PyCUDA and JCUDA adopt the technique of embedding the source code for kernel functions defined for GPU execution as strings.

To resolve the above problems, our research objective is to design, implement, and evaluate a library that supports describing kernel program by Ruby script language. Ruby is a productive script language and MasCUDA enables describing kernel program by Ruby.

\section{OVERVIEW OF MASCUDA}

MasCUDA is a Ruby library enabling the development of developing programs using the Ruby programming language. Ruby eliminates troublesome compiling and features an extensive standard library making for highly productive development. The MasCUDA library also enables the use of OpenGL [8] as a visualization function essential to MAS.

MasCUDA processing is constructed in two components: agent and space generation, and agent rule description. Agent and space generation is executed on CPU (HOST) while agent rule execution is executed on GPU (DEVICE) side. The execution steps of MasCUDA are shown in Figure 1.

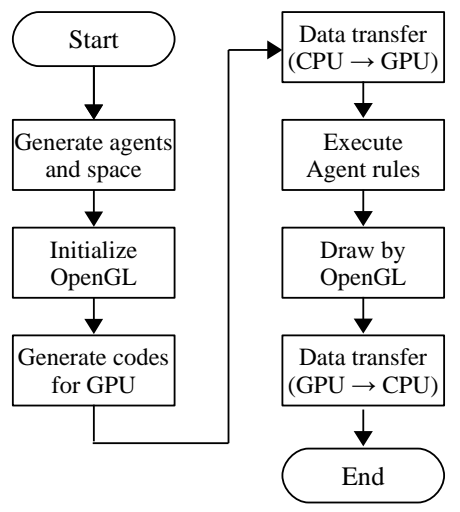

Figure 1. Execution steps of MasCUDA.

The execution begins by generating agents and space on the HOST side and initializing OpenGL for drawing. The operation of agent action is described as kernel functions executed in parallel on GPU. By using MasCUDA, users can develop these kernel functions by Ruby. To execute a program on a GPU, kernel functions described in Ruby must be converted to GPU assembly code called PTX [9]. MasCUDA generates PTX using rbcuda [10], which was developed as a domain-specific language (DSL) for CUDA by GPU. Next, the agent and space data are transferred from te CPU to GPU. Then agent actions are executed on GPU in parallel. The process then performs drawing of results and finally transfers the results of execution from GPU to CPU. In next section, we will explain rbcuda, the DSL for GPU by Ruby.

\section{RBCUDA AS DSL FOR MAS}

\subsection{Overview}

The rbcuda is a Domain Specific Language (DSL) for the use of CUDA programming framework by Ruby. It generates the PTX machine language so that programs described in Ruby can be executed by CUDA. rbcuda was developed using the LLVM compiler infrastructure [11].

In order to execute Ruby code which defines the function on the GPU must be converted to PTX assembly code for GPU execution. This PTX conversion consists of the following steps.
- Parsing the Ruby code and generate an abstract syntax tree (AST).

- Execute type inference and add type information to AST to perform type specification of PTX, a typed assembly code.

- Generate LLVM Intermediate Representation (LLVM-IR), from typed AST.

- Generate PTX from LLVM-IR using CUDA Compiler (NVCC).

\section{API PROVIDED BY MASCUDA}

MasCUDA provides following classes and APIs (Application Programming Interfaces).

\subsection{API for Agent and Space Generation}

The classes provided by MasCUDA for agent and space generation and the main methods defined in those classes are summarized below.

\subsubsection{Mas space: Class that handles space \\ - add var: Add space attribute \\ - set map: Set information for cells in space \\ 5.1.2 Mas_agent: Class that handles agents \\ - ā̄d_var: Add agent attribute \\ - typēset: Set agent attribute \\ - agent_set_cell: Place agent in space \\ - init_ogl: Initialize for drawing \\ - set_color: Set color of each agent at drawing time \\ set_pointsize: Set size of points at drawing time}

\subsection{APIs For Action Rule Description}

The main methods provided by MasCUDA for agent action are as follows.

- get_agt_Around: Get surrounding agents according base $\bar{d}$ on given parameters

- get_agtid_Around: Get surrounding agents randomly

- move: Move to the specified direction

- move maprand: Move randomly to a cell within specified range

- rand_degree: Switch direction randomly within specified range

Also provided is an API called register action for registering a method describing action rules as a kernel function.

\subsection{Description of Agent and Space Generation}

In a first step of simulation, agent and space generation is required. The first step is to generate the space in which agents will act. Programmers can generate a space by instantiating Mas_space class and specifying the size of the space, the type of model, etc.

The next step is to generate agents by instantiating Mas_agent class. At this time, programmer must specify the space for agent action, the number of agents, etc. Given an agent object created in this way, they will be placed it in the space, set agent attributes, etc. by calling various methods.

An example of describing agent and space generation is shown in Listing 1 . The first and second lines generate space generation and agent respectively. The third line arranges the agents in that space and the fourth line initializes agent attributes.

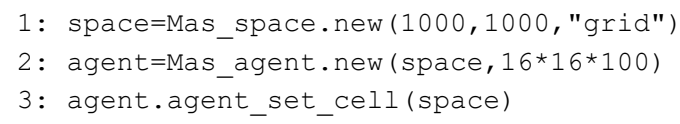




$$
\text { 4: agent.type_set }(1,2)
$$

Listing 1 Example code of space and agent generation.

\subsection{Action Rule Description}

Action rules are described as kernel functions executed on GPU. MasCUDA users develop methods that describe agent action rules and then register those methods as kernel functions. The number of threads and blocks used by a GPU are set automatically according to the number of agents and agent action is processed in parallel. Data transfer to device memory (GPU) must be performed beforehand the execution of kernel functions, but MasCUDA performs this operation automatically. At the time of kernel execution, MasCUDA transfers data from host memory (CPU) to device memory (GPU), and after kernel execution, it transfers data from device memory to host memory and releases memory automatically. Therefore, programmers do not need to make any descriptions related to memory management.

As libraries of agent action rules description, MasCUDA provides methods frequently used in MAS such as moving to a specified cell and getting surrounding agents. In order to execute kernel functions, there are important two issues; they are "agent conflict" and "synchronization".

\subsubsection{Agents Conflicts}

Processing MAS in parallel holds the possibility of agents simultaneously moving into the same cell as shown in Figure 2. This problem can be solved by the use of mutual exclusion, which is a processing that prevents accessing the same data simultaneously. CUDA enables mutual exclusion by using atomic functions, which enable a certain thread to read or write data in memory without interference from other threads.

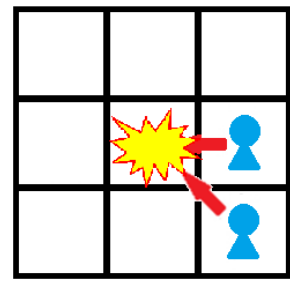

Figure 2. Agent conflict.

\subsubsection{Synchronizing Agents}

Some MAS models require the description of rules so that certain phenomena occur at the same time. For example, when obtaining agent information at a certain point in time, a failure to simultaneously execute each agent's rule generates an offset in rule execution timing thereby creating the possibility of a change in state before obtaining agent information. This is called the MAS synchronization problem. Methods for achieving synchronization include the method of performing agent actions all together at the end of each step and the method of using agent states at the immediately previous step.

CUDA does not strictly execute multiple threads simultaneously. Each GPU thread is executed asynchronously so that an offset occurs in execution order. As a result, MAS with GPU does not execute all agents simultaneously, so some sort of synchronization is necessary.

MasCUDA uses the state one step prior to the current state for synchronization. In CUDA, when synchronization is performed, a built-in function for synchronizing each thread must be called at the end of each agent rule execution step. With this method, however, the developer must be familiar with GPGPU programming, which is the reason why we adopted the method of using the state one-step-prior. Using the library which requires for synchronization will automatically create variables for storing agent states one step prior to the current state. The one-step-prior state is stored in those variables at each step and data transfer from CPU to GPU is performed automatically. The library provided by MasCUDA performs synchronization processing by using those variables.

\subsection{Example of Action Rule Description}

An example of action rule description is shown in Listing 2. The agt_step method from line 1 to line 17 describes the rule for agent action. Lines 3 and 4 each call the get_agt_Around function, which obtains the state of nearby agents. Lines 13 to 15 describes the moving the agent to another cell using the move function according to that agent's relationship with nearby agents. The move function moves the agent randomly within the specified scope. Line 18 uses the register_action function to register the agt step method as a kernel functions and line 19 invokes the agent action.

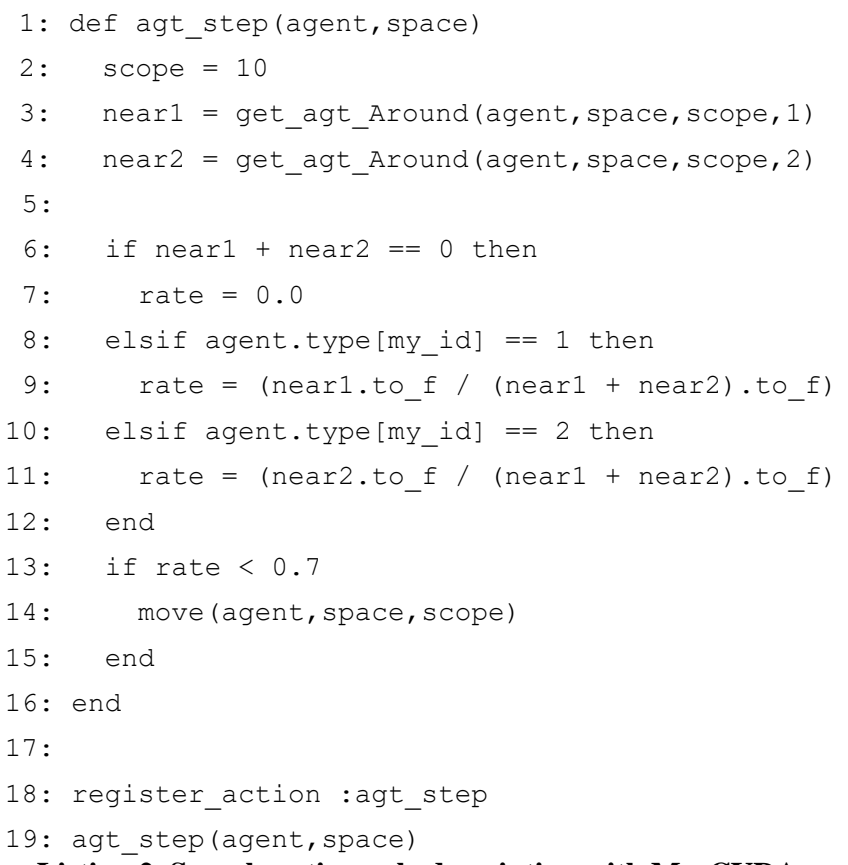

Listing 2. Sample action rule description with MasCUDA.

\section{IMPLEMENTATION AND EVALUATION OF MAS USING MASCUDA}

In this section, we describe several major MAS models using MasCUDA and demonstrate its effectiveness by applying it to models. Furthermore, to evaluate the performance and descriptiveness of MasCUDA, we implemented the same model using MasCUDA Ruby, and CUDA-C and compared execution speed and the total amount of source code.

\subsection{Model of Agent Action in Lattice Space}

This model is the most frequently seen model in MAS. This model features a lattice-shaped space with agents moving within that space. Each agent can only exist at a lattice point. As our first example of applying MasCUDA, we implemented two typical examples of this model, the segregation model [12] and Sugarscape model [13]. 
Proposed by the American economist T. C. Schelling, the segregation model explains the social phenomenon of the segregation in a MAS-like manner. This model describes how individual tendencies regarding neighbors can lead to segregation. The model is especially useful for the study of residential segregation of ethnic groups where agents represent householders who relocate in the city. This is a well-known model in the field of MAS. Simulation results for the segregation model implemented by MasCUDA are shown in Figure 3. In these results, two types of agents exist are shown in blue and green, and cells in which no agents exist are shown in black. Agents were randomly arranged at the start of simulation, but it could be observed that agents of the same type would form clusters as the simulation progressed. The number of agents is 30,000 , which is relatively larger than conventional experiment. MasCUDA could execute this large model in less than 10 seconds.
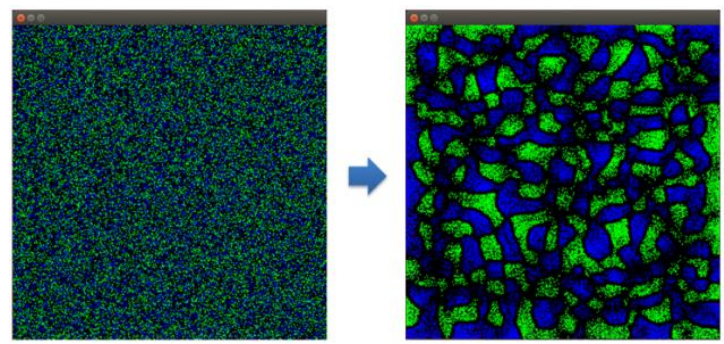

Figure 3. Segregation model by MasCUDA.

Next, the Sugarscape model is a proposed by J. M. Epstein and R. Axtell. In this model, sugar (resource) is arranged in a certain manner in model space and agents move about that space to collect more of that resource. The Sugarscape model can be extended to represent a variety of social phenomena. Simulation results for the Sugarscape model implemented by MasCUDA are shown in Figure 4. In this figure, agents are shown in red and the resource in the space in yellow. More of the resource is present at places with a darker shade of yellow. In this simulation, it could be observed that the agents would move to places with the resource.

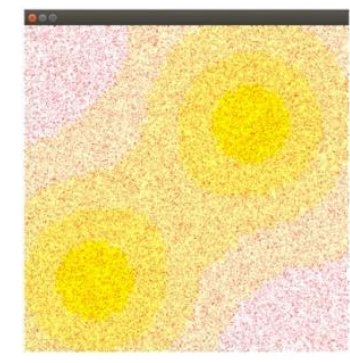

(a) Initial State

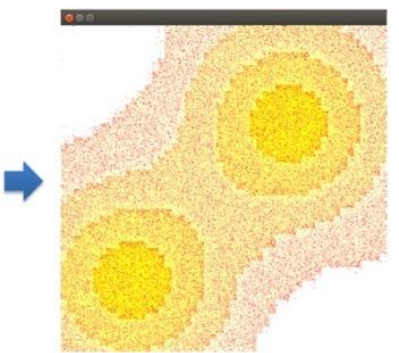

(b) After 100 steps
Figure 4. Sugarscape model in a large.

These two experiments proved the descriptive power of MasCUDA.

\subsection{Model of Cells in Lattice Space Becoming Agents}

In the type of model in 6.1, cells in lattice space exist apart from agents, but the model described here treats each cell itself as an agent in lattice space. As our second example of applying MasCUDA, we implemented two examples of this model: the forest fire model and the culture dissemination model [14].

The forest fire model simulates the spread of a fire that breaks out in a forest. Simulation results for the forest fire model implemented by MasCUDA are shown in Figure 5. In the figure, raw land is shown in white, trees in green, trees that have caught fire in red, and trees whose fires have died down in black. It could be observed from this simulation that the fire would jump to nearby trees and spread through the forest.

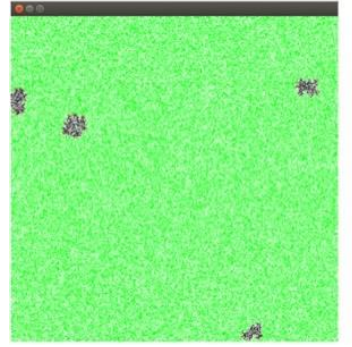

(a) Initial state

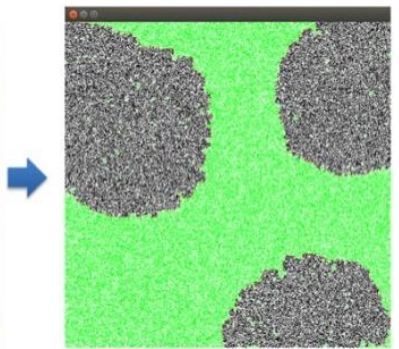

(b) After 300 steps
Figure 5. Forest fire model.

The culture dissemination model is a model proposed by the economist R. M. Axelrod for reproducing the spread of culture. A key feature of this model is that culture can be expressed by a combination of multiple attributes such as language, religion, and occupation. Simulation results for the culture dissemination model implemented by MasCUDA are shown in Figure 6. This simulation assumed that culture was made up of the three elements of language, religion, and occupation and that each of these elements came in nine types. Although agents were randomly arranged at the start of simulation, it could be observed that multiple clusters would be generated and that each cluster would expand as the simulation progressed.

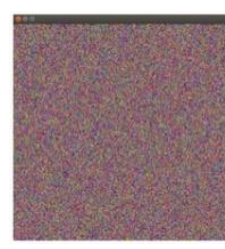

Initial state

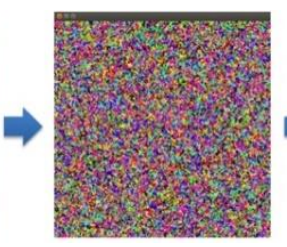

(b) After 500 steps

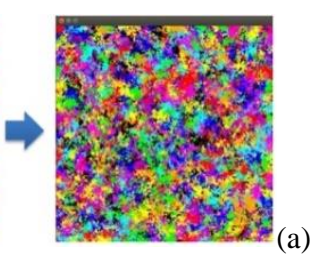

(c) After 1000 steps
Figure 6. Culture dissemination model.

\subsection{Model of Agent Action in Free Space}

In this model, agents have floating-point coordinate values as an attribute and move freely through space. As our third example of applying MasCUDA, we used it to implement the Boid model [15] and the infection model [16].

The Boid model devised by C. W. Reynolds simulates the flocking behavior of birds. The word "boid" signifies a bird-like object and stands for "bird android." Simulation results for the Boid model implemented by MasCUDA are shown in Figure 7. Here too, agents were randomly arranged at the start of simulation, but it could be observed that flocks would form over time.

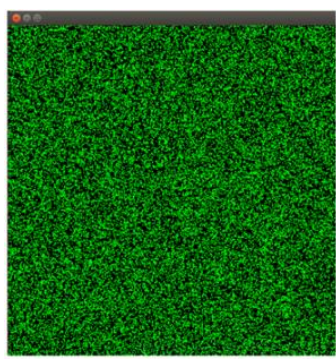

(a) Initial state

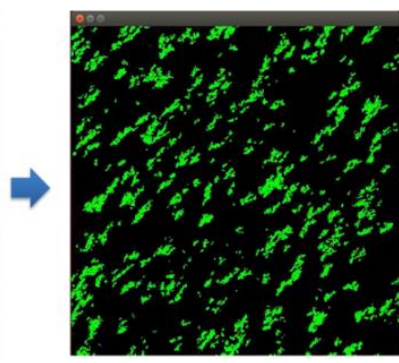

(b) Final state
Figure 7. Boid model. 
The infection model observes the epidemic process of an infection. In this model, an infection spreads when an agent becomes infected by nearby carriers or infected persons. Simulation results for the infection model implemented by MasCUDA are shown in Figure 8. In the figure, healthy persons are shown in green and carriers and infected persons are shown in pink and orange, respectively. At the start of simulation, only several carriers existed, but it could be observed that the number of carriers and infected persons would increase as the simulation progressed.

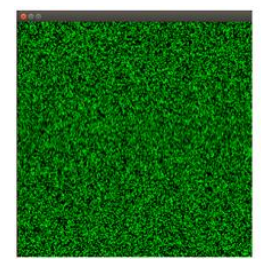

(a) Initial state

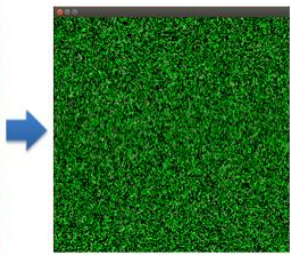

(b) 100 steps after

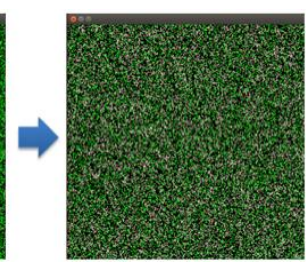

(c) 300 steps after
Figure 8. Infection model.

\section{EXPERIMENTAL EVALUATION}

We implemented all models mentioned in section 6 using MasCUDA, Ruby, and CUDA-C and compared execution speed and number of source code lines for evaluating the effectiveness of MasCUDA. In this section we will report the result of evaluation by using Segregation model as an example.

\subsection{ExecutionSpeed}

Table 1 lists the times required for agent generation and for agent action and data transfer at each step by MasCUDA, Ruby, and CUDA-C for the case of $1,000,000$ agents.

Table 1. Comparison of execution speed

\begin{tabular}{|c|c|c|c|}
\hline & $\begin{array}{c}\text { Agent and } \\
\text { space } \\
\text { Generation }\end{array}$ & $\begin{array}{c}\text { Data } \\
\text { Transfer }\end{array}$ & $\begin{array}{c}\text { Agent } \\
\text { Acton }\end{array}$ \\
\hline $\begin{array}{c}\text { Ruby } \\
\text { native }\end{array}$ & $706[\mathrm{~ms}]$ & N/A & $4371734[\mathrm{~ms}]$ \\
\hline CUDA-C & $148[\mathrm{~ms}]$ & $50.5[\mathrm{~ms}]$ & $15785[\mathrm{~ms}]$ \\
\hline $\begin{array}{c}\text { MasCUD } \\
\text { A }\end{array}$ & $1169[\mathrm{~ms}]$ & $229.7[\mathrm{~ms}]$ & $20077[\mathrm{~ms}]$ \\
\hline
\end{tabular}

In the experiment, upper limit of the number of actions was set to 100 and the agent range of view to 5 while varying the number of agents from 10,000 to $1,000,000$. The execution times of MasCUDA, Ruby, and CUDA-C are compared in Figure 9.

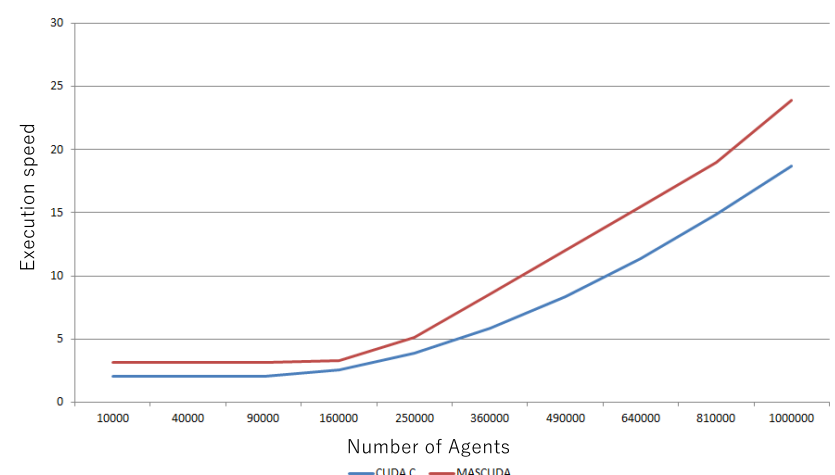

Figure 9. Comparison of execution speed of CUDA-C and MasCUDA.
Compared with MasCUDA and CUDA-C, the execution time of Ruby is overwhelmingly long, and as a result, the execution times of MasCUDA and CUDA-C are practically lying along the X-axis and overlapping each other. Therefore, execution speed of Ruby is omitted in this figure.

It can be seen From Figure 9 and Table 1 that MasCUDA is faster than Ruby as the number of agents increases. On the other hand, it can be seen from Figure 9 and Table 1 that MasCUDA is slower than CUDA-C.

\subsection{The Number of Source Code Lines}

The results of comparing the number of source code lines using the segregation model as an example are listed in Table 2. Specifically, the table lists the number of lines required to describe the processes of agent generation, memory management, agent action, and drawing.

Table 2. The number of lines of source code

\begin{tabular}{|c|c|c|c|c|}
\hline & $\begin{array}{c}\text { Agent } \\
\text { and space } \\
\text { Generatio } \\
\text { n }\end{array}$ & $\begin{array}{c}\text { Memory } \\
\text { managem } \\
\text { ent }\end{array}$ & $\begin{array}{c}\text { Agent } \\
\text { Action }\end{array}$ & $\begin{array}{c}\text { Drawin } \\
\text { g }\end{array}$ \\
\hline $\begin{array}{c}\text { Ruby } \\
\text { native }\end{array}$ & 29 & 0 & 100 & 55 \\
\hline CUDA-C & 49 & 46 & 135 & 174 \\
\hline $\begin{array}{c}\text { Mas } \\
\text { CUDA }\end{array}$ & 5 & 0 & 18 & 2 \\
\hline
\end{tabular}

These results show that MasCUDA can greatly reduce the number of source code lines. Furthermore, while a description of memory management is required in CUDA-C, no description is required in MasCUDA since memory management is performed automatically. As for description of agent action, Ruby and CUDA-C must construct that description in detail. MasCUDA, in contrast, can greatly reduce the number of lines. This is because essential operations required to describe agent actions such as get agt_Around and move are already provided by MasC $\bar{C}$ UA. Using MasCUDA also enables developing GUI in only two lines. The effects described above show that MasCUDA can reduce the number of source code lines significantly and achieve a high level of abstraction in description.

\subsection{Discussions}

\subsubsection{Execution Speed}

It can be seen from the results presented in section 7.1 that MAS can be executed at high speeds using a GPU. Compared to Ruby that performs sequential execution, MasCUDA performs parallel processing on multiple GPU threads and is consequently faster as the number of agents increase. On the other hand, MasCUDA is slower than CUDA-C. Since MasCUDA uses Ruby, an interpretive language, it is executed while the source code in sequentially interpreted. This eliminates the task of compiling and makes for high development efficiency but slows down execution speed. The results in Table 1 show that execution time for agent and space generation is approximately eight times longer by MasCUDA compared with CUDA-C, which can be largely came from the fact that Ruby is an interpreted language. Similarly, execution time for data transfer is approximately four times longer by MasCUDA than by CUDA-C. MasCUDA uses the Array class of Ruby, but to transfer such an array to a GPU, it must first be converted to an array compatible with the $\mathrm{C}$ language. This 
conversion process is performed internally, which means that extra processing is needed compared to CUDA-C.

\subsubsection{The Number of Source Code Lines}

It can be seen from the results presented in section 7.2 that the use of MasCUDA can significantly reduce the number of source code lines. This can be explained by the following three factors.

- MasCUDA provides methods frequently observed in MAS as a library. This is a factor in greatly reducing the description of agent and space generation and agent action as shown in Table 2.

- No memory management is necessary in MasCUDA. Compared to CUDA-C, Ruby performs memory management automatically making descriptions for memory allocation and release unnecessary. In addition, MasCUDA automatically performs memory management on the GPU side to execute CUDA. MasCUDA allocates memory space on the GPU side and transfers data between the CPU and GPU automatically and internally, which makes descriptions of memory management unnecessary.

- MasCUDA simplifies descriptions for drawing. OpenGL for drawing includes many descriptions needed for creating windows, writing to buffers, and processing events such as keyboard input. With MasCUDA, the developer need only specify parameters for those descriptions to satisfy the descriptions needed for drawing. Furthermore, when linking CUDA and OpenGL, a kernel function must be described for writing to a buffer mapped to CUDA, but since this kernel function is dynamically generated, the number of source code lines has been greatly reduced.

MasCUDA enables a developer to describe MAS without specialized knowledge of GPGPU. It internally defines the number of threads and blocks and performs memory management between the CPU and GPU at the time of kernel calls, which constitute descriptions unique to GPGPU. In addition, kernel functions can be described in Ruby thereby hiding GPGPUunique descriptions and resolving a past difficulty. Finally, MasCUDA incorporates mutual exclusion and synchronization processing required for parallel processing in its library so that developers can focus their efforts on describing MAS algorithms.

\section{CONCLUSION}

This article described the design and implementation of MAS library MasCUDA using GPU and the implementation of typical MAS models using this library. We showed that MasCUDA can achieve high-speed execution of MAS and can perform simulations with simple descriptions without having to worry about GPGPU programming.

At the same time, processing speed of MasCUDA is inferior to CUDA-C as discussed in the paper. Here, we can consider upgrading of the processing system or optimization of memory management as methods for improving performance. We also point out that the six MAS models to which we applied MasCUDA in this study were basic models and not simulations of real-world social phenomena. There is therefore a need in future research to apply and test MasCUDA using more complex models on a real-world scale.

\section{ACKNOWLEDGMENTS}

This research was partly supported by JSPS Grant-in-Aid for Scientific Research (KAKENHI) (C) Grant Number JP18K04623REFERENCES

\section{REFERENCES}

[1] Wilensky, U. and Rand, W. 2015. An Introduction to AgentBased Modeling, MIT Press Cambridge, MA.

[2] Cai, Y. and See, S. (eds) 2016. GPU Computing and Applications. Springer, Heidelberg, New York.

[3] Cock, S. 2012. CUDA Programming: A Developer's Guide to Parallel Computing with GPUs, Morgan Kaufmann, Waltham, MA.

[4] Jones, P. 2014. Effective Ruby: 48 Specific Ways to Write Better Ruby. Addition-Wesley Professional.

[5] https://developer.nvidia.com/pycuda

[6] https://developer.nvidia.com/pyopencl

[7] http://www.jcuda.org/

[8] Kessenich, J. and Sellers, G. and Shreiner, D. 2017. OpenGL Programming Guide, $9^{\text {th }}$ Edition, Addison-Wesley

[9] http://docs.nvidia.com/cuda/parallel-thread-execution/

[10] Nakatsuka, T. 2017. Design and Implementation of a Domain Specific Language rbcuda for GPU Parallel Processing. (in Japanese) Master's theis Doshisha University

[11] http://llvm.org/.

[12] Shelling, T. 1969. Models of Segregation, American Economic Review, Vol. 59, No. 2, pp.488-493

[13] Epstein, J. and Axtell, R. 1996. Growing artificial Societies: Social Science from the Bottom up. MIT Press

[14] Axelrod, R. 1997. The Complexity of Cooperation - Agentbased models of competition and collaboration, Princeton University Press, Princeton, NJ.

[15] Reynolds, C. 1987. Flocks, Herds, and Schools: A Distributed Behavoral Model, Computer Graphics, Vol. 21, No. 4 , pp. $25-34$

[16] Perez, L. and Dragicevic, S. 2009. An agent-based approach for modeling dynamics of contagious disease spread, International Journal of Health Geographic, Vol.8, No.1, pp 50. DOI = https://doi.org/10.1186/1476-072X-8-50 